\title{
Linguistics meets economics: Dealing with semantic variation
}

\author{
Adam D. Clark-Joseph \& Brian D. Joseph*
}

\begin{abstract}
We explore here what happens in conversation when listeners encounter variation as well as change in semantics. Working within a general Gricean framework, and in ways somewhat akin to the "Cheap Talk" model of Crawford and Sobel (1982) and the "Rational Speech Act" model of Goodman and Frank (2016), we develop here a transactional view of communicative acts, based largely on insights drawn from economics. Taking a novel perspective, we build on what happens when communication misfires rather than examining what makes for successful communication. We see this effort as a demonstration of the utility of taking an economic perspective on linguistic issues, specifically the analysis of communicative acts.
\end{abstract}

Keywords. variation; semantics; communicative act; Grice; transaction; economics

1. Introduction. Human conversation is inherently interactional, involving, minimally, two interlocutors - a speaker and a listener - who attempt to transmit information through a series of utterances taken in turns; minimally, one utterance which may or may not provoke a response. There are several approaches and even formalized models that recognize this basic structure of conversation.

Among the first such approaches, and perhaps the most influential one to date, is that of philosopher of language Herbert Paul Grice and his maxims of conversation (Grice 1975), given in (1) in our adaptation from various sources:
a. Maxim of Quality:
Be truthful.
b. Maxim of Quantity: Be spare but informative - say no more than is needed to get
c. Maxim of Relation: your message across.
d. Maxim of Manner:
Be relevant.
Be clear.

Grice's approach assumes that the interlocutors are acting in good faith to convey and receive a message. Among other similar but more formal models is that of Crawford and Sobel (1982), the so-called "Cheap Talk" model, which considers the strategic dimensions of information transmission between two agents whose incentives may not be aligned. Notably, each agent recognizes and accounts for the strategic machinations of the other.

Most recently, too, there is the Rational Speech Act (RSA) model of Frank and Goodman (2012, 2014). The RSA has been defined by Goodman and Frank (2016:819) as "a class of probabilistic model that assumes that language comprehension in context arises via a process of recursive reasoning about what speakers would have said, given a set of communicative goals", and characterized by Yuan, Monroe, Bai, and Kushman (2018) as a system in which actors in a speech act - those serving both as literal speakers and literal listeners and as pragmatic speakers and pragmatic listeners - probabilistically "recursively reason about each other's mental states to communicate".

\footnotetext{
*Authors: Adam D. Clark-Joseph, University of Illinois (adam.clarkjoseph@gmail.com) \& Brian D. Joseph, The Ohio State University (joseph.1@osu.edu).
} 
Within the context of exploring what happens in conversation when there is variation as well as change in semantics, we propose yet another interactional model, one that shares some characteristics with the three mentioned above, but is based instead on insights drawn from economics. Moreover, it starts from a different perspective; rather than exploring what makes for successful communication, it takes as its point of departure what happens when communication misfires. Although drawn from economic principles, our approach differs from the extant economic literature in that we abstract away from strategic considerations. In other words, we suppose that speakers have aligned incentives in communicating, and there is no dissembling. This extremely simple setting allows us to isolate and identify some fundamental mechanisms.

The leading issues motivating the present study are the questions in (2):

a. Can there be change in semantics?

b. Assuming an affirmative answer to (2a), what happens when such occurs?

In what follows, we address these questions, and develop a perspective on them, and especially on (2b), that draws on economic theory, ultimately viewing conversation and the extraction of meaning from conversation in transactional terms. Our two basic observations are - on the linguistics side - that some constraint must necessarily exist on meaning change, and - on the economics side - that the consequences and costs of miscommunication produce just such a constraint.

On certain rare occasions, the costs of even a minor miscommunication can be enormous, as when NASA lost its \$125 million Mars Climate Orbiter because engineers had used conflicting units of measure. More puzzling than these large but rare errors, though, is the surprising frequency with which miscommunication causes (comparatively) modest yet non-trivial losses. As a leading illustrative example, we consider semantic discord in the entrepreneurial finance world. The associated frictions have real and non-negligible costs. This bolsters our notion that we have identified a relevant and applicable constraining force on semantic change, but at the same time, it raises further questions on both the linguistic and economic fronts. Specifically, why do we see as much costly miscommunication as we do? What linguistic forces and economic mechanisms are at work? We leave these questions for future work, and concentrate here on the foundational issues set forth in (2).

2. An answer to (2a). One answer to the first question, (2a), is no, there is no semantic change per se, no change in meanings in the way that there are changes in the shape of a morpheme (e.g. adjectival suffix -lic of Old English becoming Modern English -ly) or changes in syntax (Israeli Hebrew yeš li ha sefer 'I have the book' (literally, 'there-is to-me the book') becoming for some speakers yeš li et ha sefer (literally, 'there-is to-me OBJECT.MARKER the book'). That is, starting with a view of semantics in which the meaning of a linguistic form involves a linkage between phonological material and a real-world referent, one could take the position that except in cases of newly created entities - i.e., true inventions like computers or telephones, even the wheel prehistorically — all real-world referents - i.e., all the semantic content that could be attached to phonological forms - are already present and available; ${ }^{1}$ it is just that not all are linked to particular sequences of phonemes for all speakers in all languages. ${ }^{2}$

\footnotetext{
${ }^{1}$ There is another way in which there could be change in semantics per se besides the invention of new entities. In particular, there are institutions like sports teams, such as the New York Yankees, that stay the same over time in terms of reference but change in terms of their internal content so that there are different instantiations at different times; the 1927 Yankees were not the same team as the 1989 Yankees as to their players, uniforms, etc., but in a
} 
In this view, therefore, a case like the famous shift of meaning ${ }^{3}$ for bead, from 'prayer' (first attested c. 885 and in use in this meaning up to 1554) to 'small perforated ball... used for keeping count of prayers said' (first attested 1377) and then to a more general sense of 'small perforated body, spherical or otherwise, of glass, amber, metal, wood, etc., used as an ornament, either strung in a series to form a necklace, bracelet, etc., or sewn upon various fabrics' (first attested c.1400) should not be seen as a change on the semantic level as such. The semantics, understood as the set of real-world referents, of entities to be associated with particular forms, stays the same, but there is instead a change in which referent - a prayer or a small ball or just what - is connected to a particular lexeme, a particular string of sounds.

The same holds for shifts involving content that is less concrete than that with bead. Consider the verb impeach, for instance. In the late $14^{\text {th }}$ century (c. 1380) and early $15^{\text {th }}$ century (1425), it had a general sense of "to bring a charge or accusation against; to accuse of, charge with"; that meaning gave way by no later than the second half of the $16^{\text {th }}$ century (1569) to a more specific one of "to accuse of treason or other high crime or misdemeanor (usually against the state) before a competent tribunal", and even later $\left(18^{\text {th }} / 19^{\text {th }}\right.$ centuries $)$, especially in the American context, came to refer even more specifically to accusing an elected official of a high crime or misdemeanor (as in the US Constitution ii, §4). Thus, we see the progression given in (3):

$$
\begin{aligned}
\text { impeach } & \rightarrow \text { 'accuse of wrong-doing' (in general, c. } 14^{\text {th }} \text { century) } \\
& \rightarrow \text { 'accuse of treason or high crime' }\left(\text { c. } 16^{\text {th }}\right. \text { century) } \\
& \rightarrow \text { 'accuse an elected official of a high crime' }\left(c .18^{\text {th }} / 19^{\text {th }}\right. \text { century) }
\end{aligned}
$$

Thus, in this view, what is at issue is lexical change, a change in a given lexical entry, a kind of reattachment, as it were, between a form and a referent, and not "semantic" change per se, not a change in the real-world entities that linguistic forms are attached to. This view becomes important when we turn to a consideration of variation and meaning.

3. Variation in the meaning of a lexical item. It is well established in socio-historical linguistics that one can treat variation between speakers of ostensibly the same language as evidence of change. Working from the assumption that the variation is the result of a change that one speaker or a subset of speakers have undergone, differences in details of usage between speakers is indicative of a change in the language as a whole. By simply noting the variation, one might not be able to determine the directionality of the change, i.e., whether Speaker A's feature $x$ represents the older state or Speaker B's feature $y$ does, but the fact of a change is clear.

Among the types of variation that one can observe, along with different pronunciations for words, different realizations of sounds, different syntactic constructions, and so on, there are differences between speakers as to the meanings attached to particular words/forms. Thus, to continue the present-day example from section 2, American English speakers vary as to the

\footnotetext{
certain sense they represent the same institution. Such is also the case with individuals: any of us at age 4 is different from the same individual at age 30 or age 60 . The content of the universe of real-world referents is not altered in the PRAYER to BEAD case discussed immediately below, but it is in the Yankees case.

${ }^{2} \mathrm{We}$ recognize that there is more to semantics than just lexical meaning, including scope relations, truth conditions, and the like. We focus here just on the content side of the semantics of a given linguistic form as it is a phenomenon that is more readily evident synchronically, as discussed below, and more readily studied from a diachronic perspective.

${ }^{3}$ Except where specifically noted, all definitions here, and dates of attestation and usage, are taken from the on-line version of the Oxford English Dictionary (oed.com).
} 
meaning - the real-world referent in the view espoused in section 2 - attached to the word impeach. Some see it as meaning 'formally accuse a public official of a serious wrong-doing that can lead to ouster from office' whereas others take it to mean 'formally accuse a public official of a wrong-doing and remove that person from office'. This latter sense, and the variation that speakers show, is indicated by a Quora question from 5 Sept 2018: "If President Bill Clinton was impeached, why did he not leave the office?", suggesting confusion on the part of the questioner in the face of other speakers who understood or used the word differently. There are also caveats in Wikipedia pointing in that direction: "Impeachment is the process by which a legislative body levels charges against a government official. Impeachment does not in itself remove the official definitively from office". Such caveats would not be needed if there was no variation in the use of this word.

For expository purposes for the moment, we refer here to the variation in the use of impeach, and cases like it, as "semantic variation". In that case, then, "semantic" variation reveals an important observation concerning variation in general. In particular, semantic variation between speakers is different in kind from variation in other components. That is, in cases of phonetic variation, such as [eg] vs. [ejg] for egg, or of morphological variation, such as derived noun competence vs. competency (from adjective competent) or past tense costed vs. cost, or plural octopuses vs. octopi vs. octopodes, or of syntactic variation, such as needs washed vs. needs to be washed vs. needs washing, what is at issue is really two (or more) ways of saying the same thing. It is clear that the meaning is constant across the variants and just the forms differ. Such cases therefore meet the classic definition given by Labov and others in the variationist school of sociolinguistics for a "linguistic variable". Moreover, for speakers, such variants are recognizably different ways of saying the same thing.

However, with variation in meanings (referents) attached to a form, as with impeach, it is not a case of two ways of saying the same thing but rather the saying of two different things. That is, attached to the same form there are two meanings, two sets of real-world referents or consequences, that are different, even if related. The relevance of this observation needs to be understood against the backdrop of what we refer to as a "transactional view of communication", as explained in the next section.

4. A transactional view of communication, explained. We start with the premise that language use is inherently transactional, involving an attempt at a communicative act minimally between two speakers, an attempt by one speaker to engage in a transaction by which another speaker, an interlocutor and in this case actually a listener, gains knowledge of some meaning that the first speaker wanted to convey. As noted in section 1, we assume that incentives are aligned such that strategic considerations, deception, dissembling, etc. can be ignored.

That is to say, an utterance $\left(\mathrm{U}_{\mathrm{A}}\right)$ by one speaker $\left(\mathrm{S}_{\mathrm{A}}\right)$ is designed to elicit a response of some sort by another speaker $\left(\mathrm{S}_{B}\right)$, either in the form of a rejoinder utterance $\left(\mathrm{U}_{B}\right)$ or simply an acknowledgment, tacit or overt, that the information $\mathrm{S}_{\mathrm{A}}$ has conveyed in $\mathrm{U}_{\mathrm{A}}$ has been taken in. ${ }^{4}$ If, in such a transaction, $S_{A}$ uses a word that $S_{B}$ has never heard or uses a recognizable word in a way that $S_{B}$ has never heard it used, then $S_{B}$ will not necessarily be able to understand the message that $\mathrm{S}_{\mathrm{A}}$ is trying to convey, introducing a disruption into the exchange and thus disruption in communication and a blocking of the effectiveness of the transaction.

\footnotetext{
${ }^{4}$ We adopt this notation with apologies to economist readers and emphasize that we are not using $\mathrm{U}_{\mathrm{A}}$ and $\mathrm{U}_{\mathrm{B}}$ to denote utilities or utility functions.
} 
This transactional view offers a dimension to analyzing a communicative exchange that is economic in nature. That is, with any miscommunication, there is opportunity cost, minimally, the cost (in time and energy) of repeating oneself or negotiating with one's interlocutor to get the intended message across, since one could be doing something else if repetition/negotiation were not needed. In ordinary conversation, the costs of such miscommunications are generally minimal, especially in terms of time, even if real, perhaps at best a minor annoyance. But there can be contexts in which the costs of miscommunication can actually be very large, so that the consequences of a misunderstanding due to semantic variation can be serious, as outlined in section 5 .

5. A case in point - An answer to (2b). We illustrate the potential for dire consequences of semantic variation resulting in a disruption in the success of a communicative act by looking at potential interactions between venture capitalists (VCs) and entrepreneurs. The starting assumptions of the interlocutors are clear: entrepreneurs seek funding from VCs and VCs seek good investments. Communication is very costly in real terms, involving low-bandwidth, and it is rather decentralized. What we have in mind here is face-to-face meetings, selected on the basis of brief email exchanges, meetings which entail significant monetary costs (travel, etc.) and considerable opportunity cost in that entrepreneurs and VCs alike could be pursuing other leads. Of course, some search-friction is inevitable, but miscommunications exacerbate the costs, in the case, for instance, of face-to-face meetings that turn out to be a waste of both parties' time. ${ }^{5}$ Anecdotally, such situations are surprisingly common. ${ }^{6}$

For instance, if a VC states as their avowed goal that they are interested in finding a unicorn, in the current sense of 'highly successful start-up', that would seem reasonable and entrepreneurs might act accordingly and contact said VC if they thought they had a start-up that fit that description. It would be a waste of the entrepreneurs' time if the VC meant s/he was literally chasing the mythical one-horned beast, and the entrepreneurs interpreted unicorn differently from what the VC intended. ${ }^{7}$

Admittedly, this unicorn scenario may seem far-fetched, and to be sure, unicorn probably has not caused significant confusion, but it makes the point about possible consequences of a

\footnotetext{
${ }^{5}$ Our simplifying assumption of truthfulness in this setting with capital-hungry entrepreneurs is not nearly as tenuous as it might at first appear to be. At the phase of lengthy, detailed interaction, VCs will only be fooled by the most elaborate and meticulous web of lies. Under the mild assumption that few entrepreneurs attempt massive fraud, there will typically be little reason for an entrepreneur to lie with the aim of getting a follow-up meeting that will be useless.

${ }^{6}$ Author Clark-Joseph was a co-founder of the medical company Valisure LLC, and these anecdotes are drawn from his half-decade spent in the world of entrepreneurial and venture finance, and from his associates in that world.

${ }^{7}$ In case evidence of this start-up-related meaning is needed, we can point to the results of a google search of 28 December 2019: of the seven most likely auto-completions in a search asking just "how many unicorns", four pertained to the mythical beast ("how many unicorns ... are left in the world/are there in the ark/are left/are in the ark"), two were ambiguous ("how many unicorns ... does the us have/are in the us"), and one was clearly to be understood in the start-up sense ("how many unicorns ... are profitable"). There is also a recent coinage decacorn, meaning "a relatively new company that is worth at least $\$ 10$ billion' (https://www.dictionary.com/e/tech-science/decacorn/ search: 22 March 2020), formed on the basis of unicorn in a financial sense. We are aware, too, of a generalized novel sense of unicorn as any unique kind of entity, as witnessed by a new (as of September 2019) television show on CBS entitled "The Unicorn" about a somewhat unique type of person, namely a single male - a widower, as the storyline goes - who is "the perfect single guy, i.e., a 'unicorn': employed, attractive, and with a proven track record of commitment" (https://www.cbs.com/shows/the-unicorn/about/). It is easy to understand the development of both the financial sense and the generalized sense as metaphors based on the mythical-beast sense.
} 
disruption in communication due to a difference in the meaning attached to a particular word. ${ }^{8}$ And, semantic variation can be a real issue in the $\mathrm{VC} /$ start-up world. A more serious, and less far-fetched example involves different senses associated with different funding stages.

Some VCs focus on very early-stage start-ups, while some VCs focus on very mature startups, and other VCs focus various places in between. Two terms commonly used to characterize funding stage are angel and seed, used, for instance, to label rounds of funding (e.g., angel round). Although these terms have been around for many years, their meanings have been drifting over time. For example, over the course of two years, Clark-Joseph's firm (see footnote 6) raised three progressively larger rounds of funding, titled on the term sheets "seed", "seed v.2", and "seed v.3". Anecdotally, this drift is a significant source of miscommunication, confusion, and ultimately costly miscoordination. When an entrepreneur and a VC (unwittingly) use the word to mean different things, meetings are wasted, resources are fruitlessly expended, and meanwhile opportunities are missed.

6. On some parallels to our transactional model. In section 1, we refer to other views of communicative acts that are rather like the transactional model advocated here; in particular, Grice's maxim-based approach, the "Cheap Talk" model of Crawford and Sobel (1982), and the RSA model of Goodman and Frank (2016). ${ }^{9}$ We are certainly Gricean in our general approach, as we assume that miscommunication lurks in situations where each interlocutor believes $\mathrm{s} / \mathrm{he}$ is following Grice's maxims, so that every word that is contained in an exchange is treated as truthful, informative, relevant, and clear, and meaningful, importantly, according to each interlocutor's sense of the meaning. However, by way of drawing some distinctions between what we are advocating here and the formal models described in Section 1, we remind readers that the other models look to what makes for successful communication, whereas we focus on what can go awry in an attempt at successful communication. Moreover, those models are rooted more in game theory whereas we have more of an economic basis to our model. For instance, RSA assumes reasoning, i.e. "rational", beings, whereas we look more to the economist's notion of "a rational actor", in which actors employ beliefs and higher-order beliefs (beliefs about beliefs) their own beliefs and beliefs of others - in making decisions, and in taking action; at its most basic, "rational" here means making choices that are consistent with one's preferences, and which maximize utility.

Additionally, we focus on the transaction itself rather than the inferential recursion that in RSA, for instance, leads to success in the conveying of a message. This transactional focus

\footnotetext{
${ }^{8}$ In case a reader might think it unlikely that a $\mathrm{VC}$ and an entrepreneur in the world of start-ups and start-up funding would not be on the same page as to the meaning of a term like unicorn, we note that it is not necessarily obvious on which side of the fence the nonmythical-beast sense of unicorn originated. That is, we consider just as likely that an entrepreneur first innovatively metaphorically labelled him/herself as the "unicorn" VCs were looking for as that a VC declared him/herself to be looking for a metaphorical "unicorn" among entrepreneur candidates. Furthermore, third parties, such as reporters and analysts, may have introduced the novel usage, whereupon the divide in understanding need not align with the divide between sides of the market.

${ }^{9}$ At the time we devised our transactional model, in preparation for the presentation in an Organized Session on Formal Approaches to Grammaticalization at the Annual Meeting of the Linguistic Society of America in New Orleans (5 January 2020), we were unaware of either the "Cheap Talk" or RSA models. It was only in the session itself that RSA came to our attention and the "Cheap Talk" model came before us as we delved more deeply into RSA. We have been happy to learn of them and are pleased to be part of this general intellectual thrust toward understanding acts of communication.
} 
means that while we operate in a Gricean-based model we do so with economic sensibility, including notions such as utility and cost, added in.

7. Back to the beginning: Change in grammatical semantics. Although our goal here has been largely to introduce economic thinking into aspects of linguistic analysis especially with regard to semantic variation and change, we started with a consideration of a purely linguistic question, and so we end in the same way. The questions in (2) essentially ask what it means to talk about semantic change, and our focus has been largely on change in lexical semantics. It is reasonable to ask, therefore, whether grammatical meaning can be brought under the same view as that espoused here. In particular, do the same transactional principles - and for that matter, the computations of the RSA, for instance - apply to variation in the grammatical meaning associated with specific morphemes and periphrastic combinations? Put in other words, this question could be framed as in (4):

(4) On the first time a listener encounters a new "gram" (as a unit of encoded grammatical information), how does s/he interpret it?

We suggest that a gram is intelligible to a listener if it is semantically compositional, so that the meaning of the gram is evident from the sum of the meaning of the parts of which it is composed. However, once, or rather if, such compositionality is no longer evident, then the listener is in the position of an entrepreneur not knowing what a $\mathrm{VC}$ has in mind with a particular locution.

A related question is whether constraining "semantic" change is possible. It is reasonable to suppose that one cannot simply move from any form-meaning relation A to any other formmeaning relation $\mathrm{B}$ willy-nilly, any more than a given language $\mathrm{A}$ (where $\mathrm{A}$ is a possible human language) can turn unconstrainedly into any other possible human language (e.g. a Latin-like language turning into a Chinese-like language overnight).

We note that if nothing else, economic costs will dampen entirely unconstrained changes, but that is not a system-based constraint. However, in a world where 'prayer' can turn into 'small round glass object', due to the societal milieu of the use of rosary beads to count prayers, then are any constraints possible? ${ }^{10}$

One can ask if it matters if we are dealing with the possibly more restricted set of grammatical meanings as opposed to the quite likely less restricted set of lexical meanings (possible realworld referents). We are inclined to think that there may be no linguistic (system-based) constraints, but we leave this as an open question for now.

We hope here through this investigation of what goes on in miscommunication to have shown that there is some utility to taking an economic perspective on linguistic communicative acts. Likewise, we hope to have illustrated that linguistic phenomena merit consideration as sources and modulators of frictions in economic settings.

\section{References}

Christiansen, Bethany J. \& Brian D. Joseph. 2016. On the relationship between argument structure change and semantic change. Proceedings of the Annual Meeting of the Linguistic Society of America (PLSA) 1. 26:1-11. https://doi.org/10.3765/plsa.v1i0.3726.

\footnotetext{
${ }^{10}$ This notion is discussed in Christiansen and Joseph (2016).
} 
Crawford, Vincent P. \& Joel Sobel. 1982. Strategic information transmission. Econometrica. 50(6). 1431-1451. https://www.jstor.org/stable/1913390.

Frank, Michael C. \& Noah D. Goodman. 2012. Predicting pragmatic reasoning in language games. Science 336(6084). 998. https://doi.org/10.1126/science.1218633.

Frank, Michael C. \& Noah D. Goodman. 2014. Inferring word meanings by assuming that speakers are informative. Cognitive Psychology 75. 80-96. https://doi.org/10.1016/j.cogpsych.2014.08.002.

Goodman, Noah D. \& Michael C. Frank. 2016. Pragmatic language interpretation as probabilistic inference. Trends in Cognitive Science 20(11). 818-829. https://doi.org/10.1016/j.tics.2016.08.005.

Grice, Herbert Paul. 1975. Logic and conversation. In Donald Davidson \& Gilbert Harman (eds.), The logic of grammar. 64-75. Encino, CA: Dickenson.

Yuan, Arianna, Will Monroe, Yu Bai, \& Nate Kushman. 2018. Understanding the Rational Speech Act model. Proceedings of the 40th Annual Meeting of the Cognitive Science Society (CogSci 2018): Changing minds. 2759-2764. 